\title{
Naturally occurring and added sugar in relation to macronutrient intake and food consumption: results from a population-based study in adults
}

\author{
Niina E. Kaartinen*, Minna E. Similä, Noora Kanerva, Liisa M. Valsta, Kennet Harald and Satu Männistö \\ Department of Health, National Institute for Health and Welfare, PO Box 30, FI-00271 Helsinki, Finland
}

(Received 16 November 2016 - Accepted 16 January 2017)

Journal of Nutritional Science (2017), vol. 6, e7, page 1 of 11

doi:10.1017/jns.2017.3

Abstract

Associations between sugar intake and the remaining diet are poorly described in modern food environments. We aimed at exploring associations of high naturally occurring and added sugar intakes with sociodemographic characteristics, intake of macronutrients, fibre and selected food groups. Our data comprised 4842 Finnish adults aged 25-74 years, who participated in the population-based DIetary, Lifestyle and Genetic determinants of Obesity and Metabolic syndrome (DILGOM) study. Diet was assessed by a validated 131-item FFQ. The food item disaggregation approach was used to estimate sucrose and fructose intakes from natural sources (naturally occurring sugar) and all other sources (added sugar). Sex-specific trends in macronutrient, fibre and food group intakes across sugar type quartiles were determined with general linear modelling adjusting for age, energy intake, leisure-time physical activity, smoking, education and BMI. Overall, results were similar across sexes. Young age was found to be a determinant of higher added sugar and lower naturally occurring sugar intakes $(P<0.0001)$. High added sugar intake was associated with low fibre intake $(P<0.0001)$ accompanied with lower fruit $(P<0.0001$ women; $P=0.022$ men $)$ and vegetable consumption $(P<0.0001)$ and higher wheat consumption $(P=0.0003$ women; $P<0.0001$ men $)$. Opposite results were found for naturally occurring sugar. Butter consumption increased by $28-32 \%(P<0 \cdot 0001)$ when shifting from the lowest to the highest added sugar intake quartile, while a decrease of $26-38 \%(P<0 \cdot 0001)$ was found for naturally occurring sugar. Therefore, the associations of sugar types with dietary carbohydrate and fat quality seem opposing. Proper adjustments with dietary variables are needed when studying independent relationships between sugar and health.

Key words: Added sugar: Sucrose: Fructose: Food consumption: Adults

The significance of dietary sugars as constituents of human diets is under debate. Epidemiological studies and trials have linked high intake of sugar-sweetened beverages (SSB), one major source of dietary sugar in many countries, with poor dental health, weight gain, the metabolic syndrome, type 2 diabetes mellitus and heart disease ${ }^{(1-4)}$. This has contributed to the raised profile of sugar restriction on the public health agenda. In 2015, the WHO launched a strong recommendation to maintain the population average intake of free sugar under $10 \%$ of energy $(10 \mathrm{E} \%)$ and a conditional recommendation not to exceed $5 \mathrm{E} \%{ }^{(5)}$. In the Nordic countries added sugar is recommended to stay below $10 \mathrm{E}^{\%}{ }^{(6)}$.
When considering the health effects of dietary sugars, these are typically conceptualised as free or added sugars. By definition, both of these terms include all mono- and disaccharides that are added to foods by the manufacturer, cook or consumer and sugars naturally present in honey and syrups. Free sugars also include fruit juice and fruit juice concentrates $^{(5,7)}$. These definitions do not include intrinsic or naturally occurring sugars that are, for example, within cell walls of intact fruits, vegetables and berries. In this article we use the terms 'added sugar' and 'naturally occurring sugar' when referring to the different sugar types unless otherwise stated.

Abbreviations: DILGOM, DIetary, Lifestyle and Genetic determinants of Obesity and Metabolic syndrome; E\%, percentage energy; EI, energy intake; SSB, sugar-sweetened beverages.

* Corresponding author: N. E. Kaartinen, email niina.kaartinen@thl.fi 
It is a general concern that high (added) sugar intakes are associated with poor-quality diets. The micronutrient dilution hypothesis represents one of the most intensively studied approaches to address the effect of high sugar intake on diet quality. Several reviews have summarised that high added sugar intakes are not consistently associated with lower intakes of micronutrients ${ }^{(8)}$. This has raised the need to better characterise added sugars within the total dietary context. However, studies comprehensively reporting associations between added sugar and macronutrient intake or food consumption are scarce.

Observational studies have shown strong and consistent inverse associations between total sugars (expressed as $\mathrm{E} \%$ ) and fat intake, but the association is less clear for added or naturally occurring sugar ${ }^{(9)}$. Overall, few studies in adults have assessed the relationship between added or naturally occurring sugar and protein, alcohol or fibre. In a cross-sectional study among 11626 Scottish adults extrinsic sugars were inversely associated with fibre intake, and intrinsic sugar positively with intakes of protein and fibre ${ }^{(10,11)}$. Comparable results were obtained in two Australian studies published in 1992 and 2003 utilising comprehensive food frequency and 24-h dietary recall data, respectively ${ }^{(12,13)}$. These studies also revealed that high added sugar consumers were likely to have a different intake pattern of many foods such as lower intakes of wholemeal bread and fruit, higher intake of nonalcoholic beverages, but meat and poultry consumption not differing according to added sugar intake level. In a study concerning 14709 Americans, daily servings of meat, poultry and fish decreased with increasing added sugar level $(\mathrm{E} \%)^{(14)}$. Therefore, high added sugar intake may compromise dietary quality, which is not necessarily shared by naturally occurring sugar. Recent studies on this topic are lacking, especially studies from Northern Europe.

Given the complex nature of the modern food supply, and in order to better plan studies on sugar and health outcomes, increased understanding of the relationship between added sugars, but also naturally occurring sugar, and the overall food consumption profile is needed. This information is central in refining sugar-related communication in society.

Our objective was to construct two distinct sugar exposures on the basis of their food sources: sucrose and fructose from natural sources (fruits, berries, $100 \%$ fruit juice and vegetables; naturally occurring sugar) and added sugar sources (all other sources; added sugar). We aimed at exploring potential associations between sociodemographic and lifestyle characteristics and sugar intake in Finnish adults. Furthermore, we investigated the cross-sectional relationship of these two sugar exposures with macronutrient and fibre intakes, as well as food consumption.

\section{Subjects and methods}

Participants of the Dletary, Lifestyle and Genetic determinants of Obesity and Metabolic syndrome study

The DIetary, Lifestyle and Genetic determinants of Obesity and Metabolic syndrome (DILGOM) study was conducted in the framework of the National FINRISK study, which is a Finnish population-based chronic disease risk factor monitoring survey carried out at 5-year intervals since $1972^{(15)}$. In 2007, a random sample of 10000 people aged 25-74 years from the population register was drawn stratifying according to geographical area, sex and 10-year age groups. The subjects received an invitation to a health examination and a self-administered health questionnaire via mail. A total of 6258 subjects (participation rate: $63 \%$ participated and returned the questionnaire. All FINRISK 2007 participants were invited to the DILGOM study ${ }^{(16)}$. Of the invited, 5024 subjects (participation rate: 84 $\%$ participated in the DILGOM study visit in April-June 2007. During the study visit subjects completed a FFQ and other questionnaires on health-related behaviour. Furthermore, the subjects underwent a detailed health examination.

\section{Diet}

Food consumption was measured using a comprehensive 131-item semi-quantitative FFQ inquiring into the average use frequencies of central food groups during the previous 12 months ${ }^{(17)}$. Based on the National FINDIET 2007 Survey data ${ }^{(18)}$, each FFQ item was aggregated from 1-8 most commonly consumed foods (encoded in the food composition database) and the portion sizes were fixed sex-specifically. On the form, the portion size was specified in natural units (e.g. glass, slice) whenever possible. There were nine frequency categories for all of the items ranging from never or seldom to more than six times per $\mathrm{d}$. The FFQ has been validated against 6-d food records in 510 DILGOM study subjects ${ }^{(17)}$. Between-method correlations for carbohydrate fractions used in the present analysis ranged from 0.39 (fructose, women) to 0.67 (dietary fibre, men). Using the same validation data and methodology ${ }^{(17)}$ between-method correlations for energy-adjusted fat were 0.45 and 0.38 and for protein 0.36 and 0.42 for men and women, respectively. For food groups used in the present analysis, the crude correlations ranged in men from 0.33 (meat and meat products) to 0.69 (milk and milk products) and in women from 0.30 (sugar-sweetened juice) to 0.75 (alcoholic beverages).

\section{The food composition database and calculation of sugar intake}

The average daily consumption of foods $(g / d)$ and the intake of energy $(\mathrm{kJ} / \mathrm{d})$ and nutrients $(\mathrm{g} / \mathrm{d})$ were calculated using in-house software and the Finnish National Food Composition Database (Fineli $^{\circledR}$ ), which is in part publicly available on the Internet ${ }^{(18)}$. The database is continuously updated, and includes both basic ingredients and composite foods (foods prepared at home or processed by the food industry and catering services) with individual recipes. Analysed values of nutrients are mainly based on Finnish studies. Data from the food industry and international, mainly Nordic, tables have also been used.

Due to the lack of added sugar values in the database, a food disaggregation procedure was applied to yield nutrient values at the basic ingredient level (Fig. 1). For example, according to the database recipe and predefined retention factors, sweet apple tart was disaggregated to wheat flour, butter, apples and table sugar. These foods represent the ingredient-level groups 'wheat', 'butter and butter mixtures', 'fruits' and 'sugar and syrups'. Using this procedure, all sucrose from the ingredient 


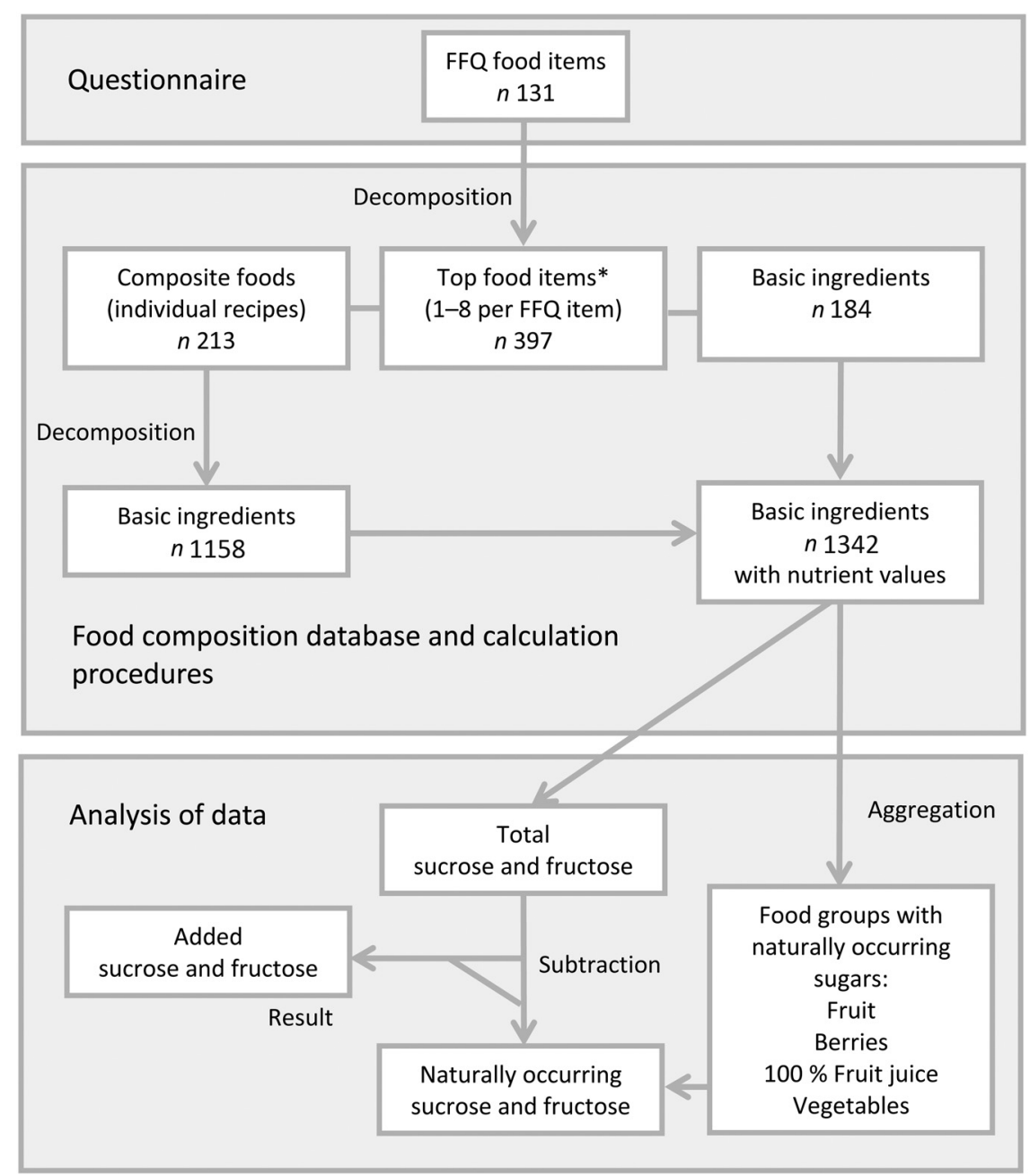

Fig. 1. Decomposition of FFQ food items to basic ingredients using the Finnish national food composition database (Fineli $\left.{ }^{\circledR}\right)^{(18)}$ and aggregation of basic ingredients in order to calculate naturally occurring and added sugars. * Most consumed foods per FFQ food item based on the National FINDIET 2007 Survey ${ }^{(18)}$.

groups 'fruits', 'berries', ‘100\% fruit juices' and 'vegetables' were summed up to yield the naturally occurring sucrose variable. The added sucrose variable was formed by subtracting the naturally occurring sucrose from total sucrose. Overall, the same procedures were applied for fructose.

For the analysis of associations between naturally occurring and added sugar intakes and food consumption we selected food groups illustrating both added sugar sources (sugars and syrups, sweet bakery products, sweets and chocolate, and SSB) and naturally occurring sugar sources (fruits, berries, $100 \%$ fruit juices and vegetables). To attain an all-round view of the diets, we also included food groups illustrating the intake of fibre (rye $v$. wheat), starch (potatoes and potato products), protein and fat (meat, fish, milk products, butter and vegetable margarine) and stimulants (coffee, tea and alcoholic beverages).

\section{Sociodemographic and lifestyle variables and anthropometric measures}

During the health examinations, trained research staff measured height $(\mathrm{cm})$, weight $(\mathrm{kg})$ and weight circumference $(\mathrm{cm})$ according to standardised protocols ${ }^{(19)}$. BMI $\left(\mathrm{kg} / \mathrm{m}^{2}\right)$ was computed as weight $(\mathrm{kg})$ divided by squared height $(\mathrm{m})$.
Questionnaires inquired about the subjects' education, leisure-time physical activity and smoking status. To adjust for the increase of average school years and the extension of the basic education system over time, total years of education were used to classify subjects into three educational levels (low, middle and high) according to birth year. Leisure-time physical activity was computed as a categorical variable with three levels: inactive (mainly light activities, e.g. reading, watching television), moderately active (e.g. walking, cycling or gardening at least $4 \mathrm{~h}$ per week) or active (physically demanding activities, e.g. running, cross-country skiing or swimming at least $3 \mathrm{~h}$ per week). Smoking status was defined by using three categories: never smoker, quit, and current smoker.

\section{The analytical sample}

After exclusion of subjects with a missing FFQ ( $n$ 28), a totally or partly empty FFQ ( $n$ 74), extreme high or low energy intake (EI) corresponding to $0.5 \%$ at both ends of the energy-intake distribution in each sex ( $n$ 48), missing BMI $(n 5)$ and pregnant women ( $n$ 27), the current study yielded a sample size of 4842 subjects. Due to sex-specific FFQ portion sizes, differences 
in the sugar intakes, and supposedly different general heath behaviour between the sexes, all analyses were performed separately for women (n 2599) and men (n 2243).

\section{Statistical analyses}

All analyses were performed using the SAS statistical software package version 9.3 (SAS Institute Inc.). Nutrient intakes were $\log$ (natural) transformed in order to satisfy the normality assumption and subsequently adjusted for total EI using the residual method ${ }^{(20)}$. The exposure variables (naturally occurring sugar, added sugar) were divided into quartiles using sex-specific cut-offs. Demographic background data were calculated both in the whole population and by quartiles of naturally occurring and added sugar intake. To take possible misreporting of EI into account, we calculated the ratio of reported EI and predicted BMR and classified subjects as either under-reporters (EI:BMR $\leq 1 \cdot 14)$ or plausible reporters $(E I: B M R>1 \cdot 14)^{(21,22)}$. Nutrient intakes were calculated by quartiles of sugar intakes. The $P$ values for trend across quartiles were determined with general linear modelling for continuous background variables and nutrient intakes, and the $\chi^{2}$ test for binary background variables. For the trend analyses, subjects were assigned the median value of their sugar intake quartile, which was used as a continuous independent variable in the modelling. We applied two models in the linear trend analysis of nutrient intakes across sugar intake quartiles: age and EI (model 1); age, EI, leisure-time physical activity, smoking status, education and BMI (model 2). Model 2 was repeated without energy under-reporters.

The continuous food group variables were transformed according to the natural $\operatorname{logarithm} \log (x+1)$ to fulfil the model assumptions. The arithmetic means and $95 \%$ CI of the transformed variables by sugar intake quartiles were calculated and antilogarithms of these were taken to yield geometric means and $95 \%$ CI for reporting. $P$ values for trend were determined with linear regression using the median values of sugar intake quartiles as continuous independent variables and each food group as continuous dependent variable at a time. We used two different sets of adjustments: age and EI (model 1); age, EI, leisure-time physical activity, smoking status, education and BMI (model 2), which was repeated without energy under-reporters.

\section{Results}

\section{Background and other characteristics of subjects}

Basic subject characteristics are given in Table 1. The average relative intake of total sugar (fructose and sucrose) was higher

Table 1. Characteristics and nutrient intakes of subjects in the Dletary, Lifestyle and Genetic determinants of Obesity and Metabolic Syndrome study (Medians, mean values or percentages, and $95 \%$ confidence intervals)

\begin{tabular}{|c|c|c|c|c|c|c|}
\hline & \multicolumn{3}{|c|}{ Women ( $n$ 2599) } & \multicolumn{3}{|c|}{ Men ( $n$ 2243) } \\
\hline & Median & Mean or \% & $95 \% \mathrm{Cl}$ & Median & Mean or \% & $95 \% \mathrm{Cl}$ \\
\hline \multicolumn{7}{|l|}{ Characteristic } \\
\hline Age (years) & 53.0 & $52 \cdot 0$ & $51 \cdot 4,52.5$ & $55 \cdot 0$ & 53.2 & $52 \cdot 6,53 \cdot 7$ \\
\hline Physically inactive subjects (\%) & & 18.9 & $17 \cdot 4,20 \cdot 4$ & & 18.5 & $16 \cdot 9,20 \cdot 1$ \\
\hline Low educated subjects (\%) & & 31.5 & $29 \cdot 7,33 \cdot 3$ & & $27 \cdot 3$ & $25 \cdot 4,29 \cdot 1$ \\
\hline Current smokers (\%) & & 14.6 & $13 \cdot 2,16 \cdot 0$ & & 20.5 & $18 \cdot 9,22 \cdot 2$ \\
\hline Energy under-reporters (\%) & & 19.4 & $17 \cdot 9,21 \cdot 0$ & & $22 \cdot 6$ & $20 \cdot 8,24 \cdot 3$ \\
\hline BMI $\left(\mathrm{kg} / \mathrm{m}^{2}\right)$ & $25 \cdot 7$ & $26 \cdot 8$ & $26 \cdot 6,27 \cdot 0$ & $26 \cdot 6$ & $27 \cdot 2$ & $27 \cdot 0,27 \cdot 3$ \\
\hline Waist circumference $(\mathrm{cm})$ & 84.5 & $86 \cdot 8$ & $86 \cdot 3,87 \cdot 3$ & 95.5 & $96 \cdot 5$ & $96 \cdot 0,97 \cdot 0$ \\
\hline \multicolumn{7}{|l|}{ Nutrient intake $(\mathrm{g} / \mathrm{d})^{*}$} \\
\hline Carbohydrate & 284 & 284 & 282,285 & 270 & 271 & 270,273 \\
\hline Total sugar (fructose and sucrose) & 83.1 & 85.1 & $84 \cdot 1,86 \cdot 1$ & 69.9 & $72 \cdot 6$ & $71 \cdot 5,73 \cdot 7$ \\
\hline Naturally occurring sugar $\dagger$ & $32 \cdot 7$ & $36 \cdot 4$ & $35 \cdot 6,37 \cdot 1$ & $24 \cdot 2$ & $27 \cdot 8$ & $27 \cdot 1,28 \cdot 6$ \\
\hline Naturally occurring fructose & $18 \cdot 1$ & $20 \cdot 0$ & $19 \cdot 6,20.5$ & $13 \cdot 2$ & $15 \cdot 3$ & $14 \cdot 9,15 \cdot 7$ \\
\hline Naturally occurring sucrose & 14.5 & $16 \cdot 3$ & $16 \cdot 0,16 \cdot 7$ & $10 \cdot 6$ & $12 \cdot 5$ & $12 \cdot 2,12 \cdot 9$ \\
\hline Added sugar $\ddagger$ & $45 \cdot 7$ & 48.8 & $48.0,49.6$ & $42 \cdot 0$ & 44.5 & $43 \cdot 7,45 \cdot 4$ \\
\hline Added fructose & 3.9 & 4.4 & $4.3,4.5$ & 3.6 & 4.0 & $4 \cdot 0,4 \cdot 1$ \\
\hline Added sucrose & 41.5 & 44.5 & $43 \cdot 7,45 \cdot 2$ & $38 \cdot 1$ & $40 \cdot 4$ & $39 \cdot 7,41 \cdot 2$ \\
\hline Dietary fibre & $31 \cdot 3$ & $32 \cdot 1$ & $31 \cdot 7,32 \cdot 4$ & $26 \cdot 2$ & $26 \cdot 7$ & $26 \cdot 4,27 \cdot 1$ \\
\hline Protein & 102 & 103 & 102,103 & 103 & 103 & 103,104 \\
\hline Fat & 81.5 & 81.9 & $81 \cdot 4,82 \cdot 4$ & 84.1 & 83.9 & $83.4,84.5$ \\
\hline Alcohol & $2 \cdot 6$ & $5 \cdot 2$ & $4.9,5.5$ & $6 \cdot 1$ & 9.7 & $9 \cdot 2,10 \cdot 3$ \\
\hline Carbohydrate (E\%) & $50 \cdot 3$ & $50 \cdot 2$ & $50 \cdot 0,50.5$ & 47.5 & $47 \cdot 7$ & $47 \cdot 4,47.9$ \\
\hline Total sugar (E\%) & $14 \cdot 3$ & 14.6 & $14.4,14.8$ & $12 \cdot 1$ & $12 \cdot 6$ & $12 \cdot 4,12 \cdot 8$ \\
\hline Naturally occurring sugar (E\%) & 5.7 & $6 \cdot 3$ & $6 \cdot 2,6 \cdot 4$ & $4 \cdot 1$ & 4.7 & $4.6,4.9$ \\
\hline Added sugar (E\%) & $7 \cdot 8$ & $8 \cdot 3$ & $8.1,8.4$ & $7 \cdot 3$ & $7 \cdot 8$ & $7.7,8.0$ \\
\hline Dietary fibre (g/1000 kcal (4184 kJ)) & 13.4 & $13 \cdot 8$ & $13 \cdot 7,14.0$ & $10 \cdot 8$ & $11 \cdot 1$ & $11 \cdot 0,11 \cdot 3$ \\
\hline Protein (E\%) & $17 \cdot 7$ & $17 \cdot 8$ & $17 \cdot 7,17 \cdot 9$ & $17 \cdot 6$ & $17 \cdot 7$ & $17 \cdot 6,17 \cdot 8$ \\
\hline Fat (E\%) & 30.4 & $30 \cdot 6$ & $30.4,30 \cdot 7$ & $31 \cdot 7$ & $31 \cdot 7$ & $31.5,31.9$ \\
\hline Alcohol (ethanol) (E\%) & 0.7 & 1.4 & $1.3,1.5$ & 1.9 & 2.9 & $2 \cdot 8,3 \cdot 1$ \\
\hline
\end{tabular}

$\mathrm{E} \%$, percentage energy.

${ }^{*}$ Nutrient intake was adjusted for total energy intake using the residual method ${ }^{(20)}$

† Naturally occurring sugar is defined as all sucrose and fructose naturally present in fruits, berries, vegetables and $100 \%$ fruit juice used as such or as a food ingredient. $\ddagger$ Added sugar is defined as sucrose and fructose originating from all other foods and food ingredient sources as the above mentioned, and was calculated as (total sucrose + fructose) - (naturally occurring sugar). 
in women compared with men. On average, $43 \%$ of women's sucrose and fructose was naturally occurring, whereas the corresponding proportion for men was $38 \%$. In both sexes, sucrose covered on average $45 \%$ of the naturally occurring sugar and $91 \%$ of the added sugar (Table 1).

The median naturally occurring sugar intakes across quartiles ranged from 2.9 to $10.2 \mathrm{E} \%$ in women and from 1.9 to $8.0 \mathrm{E} \%$ in men. Corresponding ranges for added sugar were from 4.7 to $12.1 \mathrm{E} \%$ in women and from 4.1 to 11.8 $\mathrm{E} \%$ in men (data not shown). In women, the increasing relative intake of naturally occurring sugar was associated with increasing age and decreasing BMI and waist circumference, whereas the proportions of physically inactive, low educated, currently smoking and energy under-reporting women appeared lower with increasing intakes (Table 2). Similar results were found in men, except that the association between increasing intake of naturally occurring sugar was not associated with BMI and waist circumference. In both sexes, the increasing relative intake of added sugar was associated with decreasing age, waist circumference and BMI (men only). No statistically significant association was found between increasing intake of added sugar and the proportions of physically inactive and low educated subjects in either sex. A significantly lower proportion of current smokers appeared with increasing relative intake of added sugar in men, but not women.

\section{Macronutrients and fibre}

In both sexes, the increasing relative intakes of both naturally occurring and added sugar were associated with higher intakes of carbohydrate and total sugar (sucrose and fructose), and lower intakes of protein, fat and alcohol ( $P$ values for trend $<0 \cdot 0001$, Table 3 , model 2). The relative intake of added sugar decreased significantly with increasing naturally occurring sugar intakes $(P<0.0001$ in women and $P=0.033$ in men), and the relative intake of naturally occurring sugar decreased with increasing added sugar intakes $(P<0.0001$ in women and $P=0.011$ in men). In both sexes dietary fibre intake increased with increasing intakes of naturally occurring sugar, while the opposite was found with increasing intakes of added sugar $(P<0 \cdot 0001)$. In general, the addition of leisuretime physical activity, smoking status, education and BMI, into the model (model 2) did not affect the linear trends in nutrient intakes across sugar quartiles. The exclusion of energy under-reporters produced similar results.

\section{Food groups}

In general, the associations between the two sugar types and food consumption were similar in the two models and across sexes (Tables 4 and 5). In model 2 , increasing naturally occurring sugar intake was statistically significantly associated with

Table 2. Sociodemographic and lifestyle characteristics by lowest and highest sugar intake quartile $(Q)$ in the Dletary, Lifestyle and Genetic determinants of Obesity and Metabolic syndrome study

(Mean values or percentages, and $95 \%$ confidence intervals)

\begin{tabular}{|c|c|c|c|c|c|c|c|c|c|c|}
\hline \multirow[b]{3}{*}{ Characteristic } & \multicolumn{5}{|c|}{ Naturally occurring sucrose and fructose intake quartile* } & \multicolumn{5}{|c|}{ Added sucrose and fructose intake quartile $\dagger$} \\
\hline & \multicolumn{2}{|c|}{ Q1 (lowest) } & \multicolumn{2}{|c|}{ Q4 (highest) } & \multirow[b]{2}{*}{$P \ddagger$} & \multicolumn{2}{|c|}{ Q1 (lowest) } & \multicolumn{2}{|c|}{ Q4 (highest) } & \multirow[b]{2}{*}{$P \ddagger$} \\
\hline & $\begin{array}{c}\text { Mean or } \\
\%\end{array}$ & $95 \% \mathrm{Cl}$ & $\begin{array}{c}\text { Mean or } \\
\%\end{array}$ & $95 \% \mathrm{Cl}$ & & $\begin{array}{c}\text { Mean or } \\
\%\end{array}$ & $95 \% \mathrm{Cl}$ & $\begin{array}{c}\text { Mean or } \\
\%\end{array}$ & $95 \% \mathrm{Cl}$ & \\
\hline \multicolumn{11}{|l|}{ Women ( $n$ 2599) } \\
\hline Age (years) & $50 \cdot 9$ & $49 \cdot 8,52 \cdot 0$ & 53.8 & $52 \cdot 9,54 \cdot 8$ & $<0.0001$ & $55 \cdot 1$ & $54 \cdot 2,56 \cdot 0$ & $50 \cdot 0$ & $48 \cdot 9,51 \cdot 1$ & $<0.0001$ \\
\hline $\begin{array}{l}\text { Physically inactive } \\
\text { subjects (\%) }\end{array}$ & $27 \cdot 5$ & $24 \cdot 0,30 \cdot 9$ & $14 \cdot 7$ & $12 \cdot 0,17 \cdot 5$ & $<0.0001$ & $18 \cdot 5$ & $15 \cdot 5,21 \cdot 5$ & $21 \cdot 5$ & $18 \cdot 3,24 \cdot 7$ & 0.26 \\
\hline Low educated subjects (\%) & 37.3 & $33.5,41.0$ & 25.9 & $22 \cdot 5,29 \cdot 3$ & 0.0001 & 31.6 & $28 \cdot 0,35 \cdot 2$ & $35 \cdot 0$ & $31 \cdot 3,38 \cdot 7$ & 0.12 \\
\hline Current smokers (\%) & $20 \cdot 2$ & $17 \cdot 1,23 \cdot 3$ & 13.4 & $10 \cdot 8,16 \cdot 0$ & $<0.0001$ & 17.5 & $14 \cdot 6,20.5$ & 13.4 & $10 \cdot 8,16 \cdot 1$ & 0.11 \\
\hline BMI $\left(\mathrm{kg} / \mathrm{m}^{2}\right)$ & $27 \cdot 2$ & $26 \cdot 8,27 \cdot 7$ & $26 \cdot 6$ & $26 \cdot 2,27 \cdot 0$ & 0.038 & $27 \cdot 2$ & $26 \cdot 8,27 \cdot 6$ & $26 \cdot 5$ & $26 \cdot 1,27 \cdot 0$ & 0.063 \\
\hline Waist circumference $(\mathrm{cm})$ & 88.2 & $87 \cdot 1,89.3$ & $86 \cdot 1$ & $85 \cdot 2,87 \cdot 1$ & 0.011 & 88.4 & $87.4,89.4$ & $85 \cdot 8$ & $84 \cdot 7,86 \cdot 8$ & 0.003 \\
\hline $\begin{array}{l}\text { Energy under-reporters } \\
(\%) \S\end{array}$ & 23.6 & $20 \cdot 3,26 \cdot 8$ & $18 \cdot 6$ & $15 \cdot 6,21 \cdot 6$ & 0.001 & $22 \cdot 8$ & $19 \cdot 6,26 \cdot 0$ & $19 \cdot 2$ & $16 \cdot 2,22 \cdot 3$ & 0.022 \\
\hline \multicolumn{11}{|l|}{ Men ( $n$ 2243) } \\
\hline Age (years) & $52 \cdot 6$ & $51 \cdot 5,53.7$ & $54 \cdot 3$ & $53 \cdot 2,55 \cdot 5$ & 0.007 & 54.5 & $53.4,55 \cdot 6$ & $52 \cdot 2$ & $51 \cdot 1,53 \cdot 3$ & 0.003 \\
\hline $\begin{array}{l}\text { Physically inactive } \\
\text { subjects (\%) }\end{array}$ & $24 \cdot 9$ & $21 \cdot 3,28 \cdot 5$ & $14 \cdot 0$ & $11 \cdot 1,16 \cdot 8$ & $<0.0001$ & $21 \cdot 8$ & $18 \cdot 4,25 \cdot 2$ & $17 \cdot 1$ & $13 \cdot 9,20 \cdot 2$ & 0.14 \\
\hline Low educated subjects (\%) & 38.1 & $34 \cdot 1,42 \cdot 1$ & $20 \cdot 8$ & $17 \cdot 4,24 \cdot 2$ & $<0.0001$ & $25 \cdot 0$ & $21 \cdot 4,28 \cdot 6$ & $30 \cdot 6$ & $26 \cdot 8,34.5$ & 0.11 \\
\hline Current smokers (\%) & 29.4 & $25 \cdot 6,33.2$ & $15 \cdot 9$ & $12 \cdot 9,19 \cdot 0$ & $<0.0001$ & $24 \cdot 3$ & $20 \cdot 8,27.9$ & $18 \cdot 1$ & $14 \cdot 9,21 \cdot 3$ & 0.041 \\
\hline BMI $\left(\mathrm{kg} / \mathrm{m}^{2}\right)$ & $27 \cdot 7$ & $27 \cdot 3,28 \cdot 1$ & $27 \cdot 4$ & $27 \cdot 1,27 \cdot 8$ & 0.59 & $27 \cdot 8$ & $27 \cdot 4,28 \cdot 1$ & $26 \cdot 4$ & $26 \cdot 1,26 \cdot 7$ & $<0.0001$ \\
\hline Waist circumference $(\mathrm{cm})$ & 98.2 & $97 \cdot 1,99 \cdot 3$ & $97 \cdot 1$ & $96 \cdot 1,98 \cdot 1$ & 0.33 & 98.5 & $97.4,99.5$ & $94 \cdot 2$ & $93.3,95 \cdot 1$ & $<0.0001$ \\
\hline $\begin{array}{l}\text { Energy under-reporters } \\
(\%) \S\end{array}$ & $29 \cdot 3$ & $25 \cdot 5,33 \cdot 1$ & $21 \cdot 6$ & $18 \cdot 2,25 \cdot 0$ & $<0.0001$ & $27 \cdot 5$ & $23 \cdot 8,31 \cdot 2$ & $21 \cdot 2$ & $17 \cdot 8,24 \cdot 6$ & 0.013 \\
\hline
\end{tabular}

* Naturally occurring refers to sucrose and fructose from fruits, berries, $100 \%$ fruit juice and vegetables. The variable is energy adjusted ${ }^{(20)}$ and divided into sex-specific quartiles. The median values and ranges of quartiles are: Q1, $16.6 \mathrm{~g} / \mathrm{d}, 0.78-21.9 ; \mathrm{Q} 2,27.3 \mathrm{~g} / \mathrm{d}, 22.0-32.6 ; \mathrm{Q} 3,38.7 \mathrm{~g} / \mathrm{d}, 32.7-46.8 ;$ Q4, 58.5 g/d, 46.9-205 (women); and Q1, 11.3 g/d, 0.33-15.2; Q2, $19.7 \mathrm{~g} / \mathrm{d}, 15.3-24.1 ;$ Q3, $29.2 \mathrm{~g} / \mathrm{d}, 24.2-36.7 ;$ Q4, $46.7 \mathrm{~g} / \mathrm{d}, 36.8-165$ (men).

† The added sucrose and fructose variable was calculated as (total sucrose + fructose) - (naturally occurring sucrose + fructose). The variable is energy adjusted ${ }^{(20)}$ and divided into sex-specific quartiles. The median values and ranges of quartiles are: Q1, 27.6 g/d, 6.2-34.7; Q2, 40.1 g/d, 34.7-45.7; Q3, 51.8 g/d, 45.7-59.5; Q4, 70.9 g/d, 59.6-190 (women); and Q1, $23.7 \mathrm{~g} / \mathrm{d}, 3.8-30.8 ;$ Q2, 36.2 g/d, 30.9-41.9; Q3, 47.3 g/d, 42.0-54.1; Q4, $66.4 \mathrm{~g} / \mathrm{d}, 54 \cdot 2-215$ (men).

$\ddagger P$ values for linear trend across quartiles were determined with generalised linear modelling for continuous background variables and the $\chi^{2}$ test for binary background variables. The quartile median values of sucrose + fructose intakes were used as continuous independent variables in the linear modelling.

$\S$ Energy under-reporters were defined based on the Goldberg cut-off value $(\leq 1 \cdot 14)$ for the ratio of reported energy intake:predicted BMR ${ }^{(21,22)}$. 
Table 3. Nutrient intakes by lowest and highest sugar intake quartile (Q) in the Dletary, Lifestyle and Genetic determinants of Obesity and Metabolic syndrome study (Mean values and $95 \%$ confidence intervals)

\begin{tabular}{|c|c|c|c|c|c|c|c|c|c|c|c|c|}
\hline \multirow[b]{3}{*}{ Nutrient intake $(\mathrm{g} / \mathrm{d}) \ddagger$} & \multicolumn{6}{|c|}{ Naturally occurring sucrose and fructose intake quartile* } & \multicolumn{6}{|c|}{ Added sucrose and fructose intake quartile $\dagger$} \\
\hline & \multicolumn{2}{|c|}{ Q1 (lowest) } & \multicolumn{2}{|c|}{ Q4 (highest) } & \multirow[b]{2}{*}{$P \S \|$} & \multirow[b]{2}{*}{$P \S \rrbracket$} & \multicolumn{2}{|c|}{ Q1 (lowest) } & \multicolumn{2}{|c|}{ Q4 (highest) } & \multirow[b]{2}{*}{$P \S \|$} & \multirow[b]{2}{*}{$P \S \mathbb{1}$} \\
\hline & Mean & $95 \% \mathrm{Cl}$ & Mean & $95 \% \mathrm{Cl}$ & & & Mean & $95 \% \mathrm{Cl}$ & Mean & $95 \% \mathrm{Cl}$ & & \\
\hline \multicolumn{13}{|l|}{ Women (n 2599) } \\
\hline Energy (MJ/d) & 9.07 & $8.82,9.31$ & 9.75 & $9.51,10.00$ & 0.0002 & $<0.0001$ & $9 \cdot 30$ & $9.05,9.55$ & 9.56 & $9.32,9.81$ & 0.06 & 0.11 \\
\hline Carbohydrate & 270 & 267,272 & 302 & 299,304 & $<0.0001$ & $<0.0001$ & 270 & 267,272 & 300 & 297, 302 & $<0.0001$ & $<0.0001$ \\
\hline Total sucrose and fructose & 68.4 & $66 \cdot 7,70 \cdot 0$ & 108 & 106,110 & $<0.0001$ & $<0.0001$ & 67.3 & $65 \cdot 6,68 \cdot 9$ & 109 & 107,110 & $<0.0001$ & $<0.0001$ \\
\hline Added sucrose and fructose & 53.1 & $51 \cdot 6,54 \cdot 7$ & 44.4 & $42 \cdot 8,45 \cdot 9$ & $<0.0001$ & $<0.0001$ & $26 \cdot 6$ & $25 \cdot 8,27 \cdot 3$ & 76.5 & $75 \cdot 8,77 \cdot 2$ & $<0.0001$ & $<0.0001$ \\
\hline Naturally occurring sucrose and fructose & $15 \cdot 8$ & $15 \cdot 1,16 \cdot 5$ & 63.2 & $62 \cdot 5,63.9$ & $<0.0001$ & $<0.0001$ & $40 \cdot 4$ & $38.9,41.9$ & $32 \cdot 7$ & $31 \cdot 2,34 \cdot 1$ & $<0.0001$ & $<0.0001$ \\
\hline Dietary fibre & 27.8 & $27 \cdot 1,28 \cdot 4$ & 36.6 & $36 \cdot 0,37 \cdot 3$ & $<0.0001$ & $<0.0001$ & 35.5 & $34 \cdot 8,36 \cdot 1$ & 28.8 & $28 \cdot 1,29 \cdot 4$ & $<0.0001$ & $<0.0001$ \\
\hline Protein & 105 & 103,106 & 99.1 & $97 \cdot 9,100$ & $<0.0001$ & $<0.0001$ & 111 & 110,112 & 93.4 & $92 \cdot 4,94.4$ & $<0.0001$ & $<0.0001$ \\
\hline Fat & 87.6 & $86.6,88.5$ & 74.9 & $73 \cdot 9,75 \cdot 8$ & $<0.0001$ & $<0.0001$ & 83.7 & $82 \cdot 7,84 \cdot 7$ & 79.6 & $78 \cdot 6,80 \cdot 6$ & $<0.0001$ & $<0.0001$ \\
\hline Alcohol & $6 \cdot 0$ & $5 \cdot 4,6 \cdot 6$ & 4.9 & $4 \cdot 3,5 \cdot 5$ & 0.09 & 0.027 & $6 \cdot 8$ & $6 \cdot 2,7.4$ & 3.8 & $3.2,4.4$ & $<0.0001$ & $<0.0001$ \\
\hline \multicolumn{13}{|l|}{ Men ( $n$ 2243) } \\
\hline Energy $(\mathrm{MJ} / \mathrm{d})$ & 11.22 & $10.89,11.56$ & 11.81 & $11 \cdot 48,12 \cdot 15$ & 0.047 & 0.05 & 11.36 & $11.02,11.69$ & 11.67 & $11.33,12.00$ & 0.22 & 0.17 \\
\hline Carbohydrate & 257 & 254,260 & 290 & 287,293 & $<0.0001$ & $<0.0001$ & 255 & 252,257 & 290 & 288,293 & $<0.0001$ & $<0.0001$ \\
\hline Total sucrose and fructose & $56 \cdot 5$ & $54 \cdot 7,58 \cdot 3$ & 93.7 & $91.9,95.5$ & $<0.0001$ & $<0.0001$ & $51 \cdot 2$ & $49 \cdot 6,52 \cdot 8$ & $98 \cdot 0$ & $96.4,99.6$ & $<0.0001$ & $<0.0001$ \\
\hline Added sucrose and fructose & 45.5 & $43 \cdot 8,47 \cdot 2$ & $42 \cdot 7$ & $41 \cdot 0,44.4$ & 0.017 & 0.033 & $22 \cdot 6$ & $21 \cdot 8,23.4$ & 71.6 & $70 \cdot 8,72 \cdot 4$ & $<0.0001$ & $<0.0001$ \\
\hline Naturally occurring sucrose and fructose & $10 \cdot 6$ & $10 \cdot 0,11 \cdot 3$ & $51 \cdot 2$ & $50.5,51.9$ & $<0.0001$ & $<0.0001$ & 28.6 & $27 \cdot 2,30 \cdot 0$ & $25 \cdot 8$ & $24 \cdot 4,27 \cdot 2$ & 0.009 & 0.011 \\
\hline Dietary fibre & 23.7 & $23 \cdot 1,24 \cdot 3$ & 30.0 & $29.4,30.6$ & $<0.0001$ & $<0.0001$ & 28.1 & $27 \cdot 5,28 \cdot 7$ & 24.9 & $24 \cdot 3,25 \cdot 6$ & $<0.0001$ & $<0.0001$ \\
\hline Protein & 105 & 104,106 & 99.7 & $98 \cdot 5,101$ & $<0.0001$ & $<0.0001$ & 110 & 109,112 & 94.5 & $93.4,95.6$ & $<0.0001$ & $<0.0001$ \\
\hline Fat & 88.6 & $87.6,89.7$ & 77.9 & $76.8,78.9$ & $<0.0001$ & $<0.0001$ & 85.4 & $84 \cdot 4,86 \cdot 5$ & 80.8 & $79.7,81.9$ & $<0.0001$ & $<0.0001$ \\
\hline Alcohol & 11.5 & $10.5,12.5$ & 8.0 & $6 \cdot 9,9.0$ & $<0.0001$ & $<0.0001$ & $13 \cdot 3$ & $12 \cdot 2,14 \cdot 3$ & 7.4 & $6 \cdot 4,8.4$ & $<0.0001$ & $<0.0001$ \\
\hline
\end{tabular}

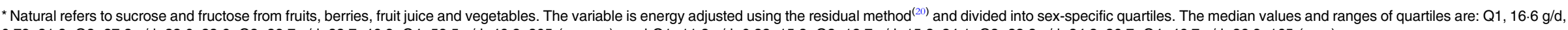
0.78-21.9; Q2, 27.3 g/d, 22.0-32.6; Q3, 38.7 g/d, 32.7-46.8; Q4, 58.5 g/d, 46.9-205 (women); and Q1, 11.3 g/d, 0.33-15.2; Q2, 19.7 g/d, 15.3-24.1; Q3, 29.2 g/d, 24.2-36.7; Q4, 46.7 g/d, 36.8-165 (men).

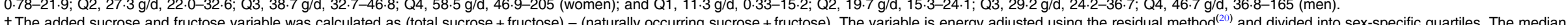

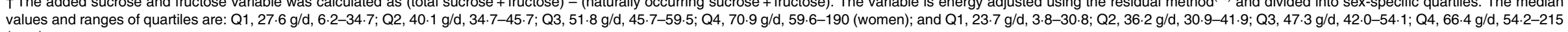
(men).

$\ddagger$ Values shown in the table are adjusted for age and total energy intake, except energy intake values are only age adjusted.

$\S P$ values for linear trends were determined with generalised linear modelling using the median values of sugar intake quartiles as continuous independent variables.

$\|$ Adjusted for age and energy intake. When energy was used as a dependent variable the analyses were adjusted for age only.

Iी Adjusted for age, energy intake, leisure-time physical activity, smoking status, education and BMI. 


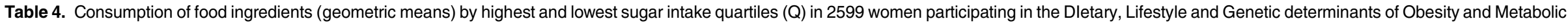
syndrome study

(Mean values and $95 \%$ confidence intervals)

\begin{tabular}{|c|c|c|c|c|c|c|c|c|c|c|c|c|}
\hline \multirow[b]{3}{*}{ Food consumption $(\mathrm{g} / \mathrm{d}) \ddagger$} & \multicolumn{6}{|c|}{ Naturally occurring sucrose and fructose intake quartile* } & \multicolumn{6}{|c|}{ Added sucrose and fructose intake quartile } \\
\hline & \multicolumn{2}{|c|}{ Q1 (lowest) } & \multicolumn{2}{|c|}{ Q4 (highest) } & \multirow[b]{2}{*}{$P \S \|$} & \multirow[b]{2}{*}{$P \S \rrbracket$} & \multicolumn{2}{|c|}{ Q1 (lowest) } & \multicolumn{2}{|c|}{ Q4 (highest) } & \multirow[b]{2}{*}{$P \S \|$} & \multirow[b]{2}{*}{$P \S \uparrow$} \\
\hline & Mean & $95 \% \mathrm{Cl}$ & Mean & $95 \% \mathrm{Cl}$ & & & Mean & $95 \% \mathrm{Cl}$ & Mean & $95 \% \mathrm{Cl}$ & & \\
\hline Sugars and syrups & $13 \cdot 1$ & $12 \cdot 5,13 \cdot 7$ & $10 \cdot 7$ & $10 \cdot 1,11 \cdot 2$ & $<0.0001$ & $<0.0001$ & 7.4 & $7 \cdot 1,7 \cdot 8$ & $18 \cdot 3$ & $17.5,19.0$ & $<0.0001$ & $<0.0001$ \\
\hline Sweet bakery products & 44.9 & $41 \cdot 7,48.5$ & $26 \cdot 7$ & $24 \cdot 7,28 \cdot 9$ & $<0.0001$ & $<0.0001$ & $22 \cdot 4$ & $20 \cdot 7,24 \cdot 1$ & $50 \cdot 3$ & $46 \cdot 8,54 \cdot 2$ & $<0.0001$ & $<0.0001$ \\
\hline Sweets and chocolate & $11 \cdot 2$ & $10 \cdot 4,12 \cdot 2$ & 9.6 & $8.8,10.5$ & 0.005 & 0.0007 & 6.0 & $5 \cdot 6,6 \cdot 6$ & $16 \cdot 6$ & $15 \cdot 4,17 \cdot 8$ & $<0.0001$ & $<0.0001$ \\
\hline Sugar-sweetened beverages & $28 \cdot 1$ & $24 \cdot 3,32 \cdot 6$ & $16 \cdot 9$ & $14 \cdot 6,19 \cdot 7$ & $<0.0001$ & $<0.0001$ & 6.8 & $5 \cdot 8,7.9$ & 71.8 & $62 \cdot 9,81 \cdot 8$ & $<0.0001$ & $<0.0001$ \\
\hline Fruits & $60 \cdot 2$ & $57 \cdot 2,63 \cdot 3$ & 398 & 379,419 & $<0.0001$ & $<0.0001$ & 193 & 180,208 & 141 & 131,151 & $<0.0001$ & $<0.0001$ \\
\hline Berries & 25.9 & $24 \cdot 3,27 \cdot 7$ & 34.7 & $32.5,37.0$ & $<0.0001$ & $<0.0001$ & 27.5 & $25 \cdot 7,29 \cdot 4$ & 34.0 & $31 \cdot 9,36 \cdot 4$ & $<0.0001$ & 0.0002 \\
\hline Fruit juices (100 \%) & 9.7 & $8.4,11 \cdot 3$ & 83.5 & $73.1,95.4$ & $<0.0001$ & $<0.0001$ & $26 \cdot 1$ & $22 \cdot 5,30 \cdot 3$ & $26 \cdot 7$ & $23.0,30.9$ & 0.88 & 0.58 \\
\hline Vegetables & 214 & 205,223 & 341 & 327,355 & $<0.0001$ & $<0.0001$ & 339 & 325,354 & 234 & 224,244 & $<0.0001$ & $<0.0001$ \\
\hline Rye & 51.6 & $48.5,54.9$ & $45 \cdot 4$ & $42 \cdot 7,48 \cdot 3$ & 0.001 & $<0.0001$ & $59 \cdot 2$ & $55 \cdot 6,62 \cdot 9$ & $41 \cdot 3$ & $38.9,44.0$ & $<0.0001$ & $<0.0001$ \\
\hline Wheat & $65 \cdot 1$ & $62 \cdot 9,67.5$ & 47.6 & $45 \cdot 9,49 \cdot 3$ & $<0.0001$ & $<0.0001$ & $52 \cdot 1$ & $50 \cdot 2,54 \cdot 0$ & $59 \cdot 0$ & $56 \cdot 9,61 \cdot 1$ & $<0.0001$ & 0.0003 \\
\hline Potatoes and potato products & 110 & 106,115 & 91.4 & $87 \cdot 6,95 \cdot 4$ & $<0.0001$ & $<0.0001$ & 108 & 103,113 & $92 \cdot 7$ & $88.8,96.8$ & $<0.0001$ & $<0.0001$ \\
\hline Meat and meat products & 140 & 134,147 & 118 & 113,124 & $<0.0001$ & $<0.0001$ & 144 & 137,150 & 111 & 106,116 & $<0.0001$ & $<0.0001$ \\
\hline Fish, fish products and shellfish & 31.6 & $29 \cdot 7,33.6$ & $34 \cdot 1$ & $32 \cdot 1,36 \cdot 3$ & 0.17 & 0.31 & 39.8 & $37.4,42 \cdot 3$ & 28.4 & $26 \cdot 7,30 \cdot 2$ & $<0.0001$ & $<0.0001$ \\
\hline Milk and milk products & 525 & 502,548 & 433 & 415,453 & $<0.0001$ & $<0.0001$ & 450 & 431,470 & 503 & 481,525 & 0.001 & 0.004 \\
\hline Butter (including butter oil mixtures) & $6 \cdot 0$ & $5 \cdot 7,6 \cdot 3$ & 3.7 & $3.5,4 \cdot 0$ & $<0.0001$ & $<0.0001$ & 4.1 & $3.9,4 \cdot 4$ & $5 \cdot 4$ & $5 \cdot 1,5 \cdot 8$ & $<0.0001$ & $<0.0001$ \\
\hline Vegetable margarine & 7.8 & $7 \cdot 2,8 \cdot 5$ & 6.9 & $6 \cdot 3,7.5$ & 0.029 & 0.009 & 8.3 & $7 \cdot 6,9.0$ & $6 \cdot 8$ & $6 \cdot 2,7 \cdot 4$ & 0.0003 & 0.0004 \\
\hline Coffee and tea & 445 & 414,479 & 433 & 402,466 & 0.72 & 0.35 & 446 & 414,479 & 405 & 376,435 & 0.08 & 0.22 \\
\hline Alcoholic beverages & 21.6 & $18 \cdot 5,25 \cdot 1$ & 21.6 & $18 \cdot 5,25 \cdot 1$ & 0.86 & 0.50 & 31.0 & $26 \cdot 7,36 \cdot 1$ & $15 \cdot 0$ & $12 \cdot 9,17.5$ & $<0.0001$ & $<0.0001$ \\
\hline
\end{tabular}

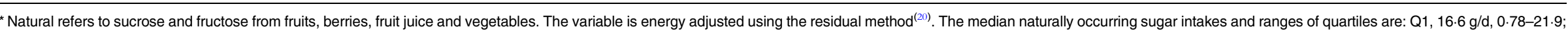
Q2, 27.3 g/d, 22.0-32.6; Q3, 38.7 g/d, 32.7-46.8; Q4, 58.5 g/d, 46.9-205.

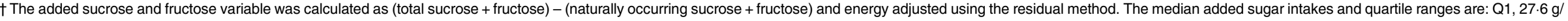
d, 6.2-34.7; Q2, 40.1 g/d, 34.7-45.7; Q3, $51.8 \mathrm{~g} / \mathrm{d}, 45 \cdot 7-59.5 ;$ Q4, 70.9 g/d, 59.6-190.

$\ddagger$ Values in the table are adjusted for age and energy intake.

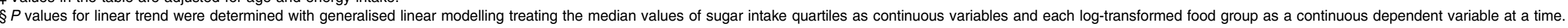

$\|$ Adjusted for age and energy intake.

ๆ Adjusted for age, energy intake, leisure-time physical activity, smoking status, education and BMI. 


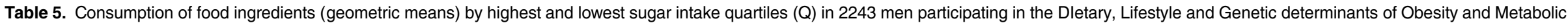
syndrome study

(Mean values and $95 \%$ confidence intervals)

\begin{tabular}{|c|c|c|c|c|c|c|c|c|c|c|c|c|}
\hline \multirow[b]{3}{*}{ Food consumption $(\mathrm{g} / \mathrm{d}) \ddagger$} & \multicolumn{6}{|c|}{ Naturally occurring sucrose and fructose intake quartile* } & \multicolumn{6}{|c|}{ Added sucrose and fructose intake quartile† } \\
\hline & \multicolumn{2}{|c|}{ Q1 (lowest) } & \multicolumn{2}{|c|}{ Q4 (highest) } & \multirow[b]{2}{*}{$P \S \|$} & \multirow[b]{2}{*}{$P \S \rrbracket$} & \multicolumn{2}{|c|}{ Q1 (lowest) } & \multicolumn{2}{|c|}{ Q4 (highest) } & \multirow[b]{2}{*}{$P \S \|$} & \multirow[b]{2}{*}{$P \S \bigoplus$} \\
\hline & Mean & $95 \% \mathrm{Cl}$ & Mean & $95 \% \mathrm{Cl}$ & & & Mean & $95 \% \mathrm{Cl}$ & Mean & $95 \% \mathrm{Cl}$ & & \\
\hline Sugars and syrups & $16 \cdot 8$ & $15 \cdot 9,17 \cdot 9$ & $14 \cdot 0$ & $13 \cdot 2,14.9$ & $<0.0001$ & $<0.0001$ & 8.4 & $7 \cdot 9,8 \cdot 8$ & $25 \cdot 4$ & $24 \cdot 2,26 \cdot 7$ & $<0.0001$ & $<0.0001$ \\
\hline Sweet bakery products & 51.8 & $47 \cdot 4,56 \cdot 6$ & 39.4 & $36 \cdot 0,43 \cdot 0$ & $<0.0001$ & $<0.0001$ & $26 \cdot 7$ & $24.5,29 \cdot 1$ & 66.4 & $61 \cdot 1,72 \cdot 2$ & $<0.0001$ & $<0.0001$ \\
\hline Sweets and chocolate & $8 \cdot 2$ & $7 \cdot 5,9 \cdot 1$ & 9.7 & $8 \cdot 9,10 \cdot 6$ & 0.047 & 0.40 & 5.4 & $4 \cdot 9,5 \cdot 9$ & 14.1 & $12 \cdot 9,15 \cdot 3$ & $<0.0001$ & $<0.0001$ \\
\hline Sugar-sweetened beverages & $33 \cdot 5$ & $28 \cdot 7,39 \cdot 0$ & $40 \cdot 4$ & $34 \cdot 7,47 \cdot 0$ & 0.21 & 0.17 & $12 \cdot 9$ & $11 \cdot 2,14 \cdot 8$ & 134 & 118,153 & $<0.0001$ & $<0.0001$ \\
\hline Fruits & 41.9 & $39.1,44.9$ & 270 & 253,289 & $<0.0001$ & $<0.0001$ & 117 & 107,128 & 104 & $94.7,113$ & 0.033 & 0.022 \\
\hline Berries & $15 \cdot 3$ & $14 \cdot 0,16 \cdot 6$ & $26 \cdot 2$ & $24 \cdot 1,28.4$ & $<0.0001$ & $<0.0001$ & $16 \cdot 0$ & $14 \cdot 8,17 \cdot 4$ & 25.4 & $23 \cdot 4,27.5$ & $<0.0001$ & $<0.0001$ \\
\hline Fruit juices (100 \%) & $14 \cdot 0$ & $12 \cdot 3,16 \cdot 0$ & 170 & 151, 192 & $<0.0001$ & $<0.0001$ & 44.6 & $38 \cdot 6,51 \cdot 4$ & 54.7 & $47.4,63.0$ & 0.07 & 0.06 \\
\hline Vegetables & 160 & 153,168 & 269 & 257,282 & $<0.0001$ & $<0.0001$ & 248 & 236,260 & 188 & 179, 197 & $<0.0001$ & $<0.0001$ \\
\hline Rye & 54.9 & $51 \cdot 4,58 \cdot 7$ & $49 \cdot 7$ & $46 \cdot 5,53 \cdot 1$ & 0.028 & 0.024 & $58 \cdot 6$ & $54 \cdot 8,62 \cdot 6$ & $46 \cdot 4$ & $43.4,49.5$ & $<0.0001$ & $<0.0001$ \\
\hline Wheat & 79.9 & $77 \cdot 1,82 \cdot 9$ & $68 \cdot 8$ & $66 \cdot 4,71 \cdot 3$ & $<0.0001$ & $<0.0001$ & $67 \cdot 4$ & $65 \cdot 0,69 \cdot 9$ & 78.0 & $75 \cdot 2,80 \cdot 8$ & $<0.0001$ & $<0.0001$ \\
\hline Potatoes and potato products & 165 & 158, 172 & 136 & 130,142 & $<0.0001$ & $<0.0001$ & 167 & 160,175 & 142 & 136,148 & $<0.0001$ & $<0.0001$ \\
\hline Meat and meat products & 204 & 196, 212 & 179 & 172,185 & $<0.0001$ & $<0.0001$ & 219 & 211,227 & 170 & 164,176 & $<0.0001$ & $<0.0001$ \\
\hline Fish, fish products and shellfish & 41.5 & $38.8,44.4$ & $49 \cdot 1$ & $45 \cdot 9,52 \cdot 5$ & 0.005 & $0 \cdot 10$ & 53.9 & $50.4,57.6$ & $40 \cdot 2$ & $37.6,43.0$ & $<0.0001$ & $<0.0001$ \\
\hline Milk and milk products & 595 & 564,628 & 478 & 453,504 & $<0.0001$ & $<0.0001$ & 545 & 516,575 & 532 & 504,562 & 0.47 & 0.33 \\
\hline Butter (including butter oil mixtures) & $7 \cdot 3$ & $6 \cdot 9,7 \cdot 8$ & 5.4 & $5 \cdot 1,5 \cdot 8$ & $<0.0001$ & $<0.0001$ & 5.4 & $5 \cdot 1,5 \cdot 8$ & 6.9 & $6 \cdot 4,7 \cdot 3$ & $<0.0001$ & $<0.0001$ \\
\hline Vegetable margarine & 8.9 & $8 \cdot 1,9 \cdot 8$ & 9.0 & $8.2,9.9$ & 0.93 & 0.97 & $10 \cdot 3$ & $9.4,11 \cdot 3$ & 8.1 & $7.3,8.9$ & 0.001 & 0.001 \\
\hline Coffee and tea & 462 & 430,497 & 422 & 392,454 & 0.06 & 0.12 & 425 & 395,457 & 464 & 432,500 & 0.12 & 0.017 \\
\hline Alcoholic beverages & $76 \cdot 6$ & $64 \cdot 6,90 \cdot 7$ & $62 \cdot 1$ & $52 \cdot 4,73.6$ & 0.08 & 0.009 & 101 & $85 \cdot 6,120$ & $53 \cdot 3$ & $45 \cdot 0,63 \cdot 2$ & $<0.0001$ & $<0.0001$ \\
\hline
\end{tabular}

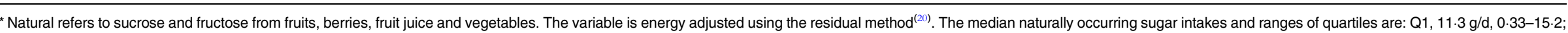
Q2, $19.7 \mathrm{~g} / \mathrm{d}, 15 \cdot 3-24 \cdot 1 ;$ Q3, $29 \cdot 2 \mathrm{~g} / \mathrm{d}, 24 \cdot 2-36 \cdot 7 ;$ Q4, $46 \cdot 7 \mathrm{~g} / \mathrm{d}, 36 \cdot 8-165$.

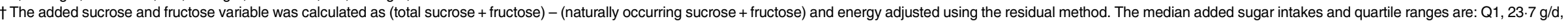
3.8-30.8; Q2, 36.2 g/d, 30.9-41.9; Q3, 47.3 g/d, 42.0-54.1; Q4, 66.4 g/d, 54.2-215.

$\mp$ Values in the table are adjusted for age and energy intake.

$\S P$ values for trend were determined with generalised linear modelling treating the median values of sugar intake quartiles as continuous variables and each log-transformed food group as a continuous dependent variable at a time.

$\|$ Adjusted for age and energy intake.

ๆ Adjusted for age, energy intake, leisure-time physical activity, smoking status, education and BMI. 
higher intakes of fruits, berries, $100 \%$ fruit juices and vegetables $(P<0.0001$, naturally occurring sugar sources), whereas the consumption of added sugar sources such as sugars and syrups, sweet bakery products, sweets and chocolate $(P=$ 0.0007 for women and NS for men), and SSB (women only, $P<0.0001)$ was lower. Compared with these results, opposite associations were found between increasing added sugar intake and the food sources of the different sugars. From the naturally occurring sugar sources only berries associated positively with rising added sugar intake $(P=<0 \cdot 0001)$, and $100 \%$ fruit juices were not significantly associated with added sugar intake in either sex. From the other food groups rye $(P<$ 0.0001 for women and $P=0.024$ for men), potatoes, meat and milk products, butter (all $P<0.0001$ for both sexes) and vegetable margarine $(P=0.009$ for women and NS for men) consumptions were lower for high naturally occurring sugar intakes. Wheat consumption decreased $14-27 \%$ from the lowest to the highest naturally occurring sugar intake quartile $(P<0.0001$ for both sexes). Fish consumption was not associated with naturally occurring sugar intake in either women or men, and alcoholic beverage consumption only among men (inverse association, $P=0 \cdot 009$ ). In both sexes, the consumption of rye, potatoes, meat, fish, vegetable margarine and alcoholic beverages decreased with higher added sugar intakes. Consumption of wheat increased 13-16\% from the lowest to the highest added sugar intake quartile $(P=0.0003$ for women and $P<0.0001$ for men). Also, the consumption of milk products $(P=0.004$ for women and NS for men) and butter $(P<0.0001$ for both sexes) increased with higher intakes of added sugar. Added sugar intake was associated also with higher coffee and tea consumption in men $(P=0.017)$ but not in women.

\section{Discussion}

This cross-sectional analysis in 4842 Finnish adults investigated associations of naturally occurring sugar and added sugar with sociodemographic characteristics and overall diet approached through macronutrient intake and consumption of selected food groups. Older age was associated with higher naturally occurring sugar intakes and lower added sugar intakes. High added sugar intake was also associated with lower fibre intake and found to potentially compromise dietary fat quality. Strengths of this study include the populationbased approach, and the use of a culturally well-adopted FFQ, which is found to be reasonably valid for measuring sucrose and fructose intakes in our study population ${ }^{(17)}$. To our knowledge, the relationship between sugar intake and different food groups in adult populations has not been comprehensively investigated in Finland or other Nordic countries before.

Overall healthier lifestyle with regard to leisure-time physical activity and smoking was associated with high naturally occurring sugar intake. These results are in line with studies showing clustering of unhealthy, but also healthy, behaviours ${ }^{(23)}$. Recently, a study in 9425 New Zealand adults demonstrated a two-level clustering of fruit and vegetable intake with physical activity, sedentary behaviour and SSB consumption: optimal wellbeing was higher in healthy behaviour combinations ${ }^{(24)}$. In our study, young age was associated with higher added sugar intakes - a phenomenon seen in other cultures as well ${ }^{(25-27)}$. This strengthens the basis to keep added sugar intake reduction on the public health agenda and focusing on total diet and total lifestyle to optimise population health.

With regard to macronutrients, our results conform to studies from the 1990s reporting an inverse relationship between sugar and fat intake (sugar-fat see-saw phenomenon) when using $\mathrm{E} \%$ of extrinsic sugar or intrinsic sugar as exposures ${ }^{(10,12,28,29)}$. Similar to our results, high relative added/ extrinsic sugar intakes have been inversely associated with protein and alcohol intakes ${ }^{(10,13)}$. In these studies, naturally occurring/intrinsic sugar was, if anything, positively associated with protein intake. In our study, rising added sugar intake was associated with a greater decrease in protein intake (14-16\% difference between highest and lowest quartile) compared with naturally occurring sugar (5-6\% difference between the highest and lowest quartiles). This suggests that added and naturally occurring sugars act as markers of divergent diets accompanied by different macronutrient compositions.

Current Nordic dietary recommendations emphasise the quality of dietary carbohydrate and fat over their amount ${ }^{(6)}$. We found a positive association between naturally occurring sugar and fibre intake, whereas the opposite was observed for added sugar. These results were independent of age, EI, leisure-time physical activity, smoking status, education and BMI. Studies from different food cultures have shown similar results. In a Scottish study utilising FFQ data from 11626 men and women aged 25-64 years, extrinsic sugar was associated negatively and intrinsic sugar positively with fibre intake after adjusting for important confounders ${ }^{(11)}$. Comparable results were also obtained using 24-h recall data in 10417 Australian adults ${ }^{(13)}$, and in 15189 Americans from the National Health and Nutrition Examination Survey (NHANES) 2003-2006 ${ }^{(30)}$. In a Dutch study, adults adhering to the dietary guideline of $<10 \mathrm{E} \%$ for added sugar scored higher for the healthy diet index components fibre, but also fruits and vegetables ${ }^{(26)}$. This is also in line with our food consumption results for fruits, berries and vegetables. Overall, results for fibre are also supported by our results for wheat and rye (to some extent). The decrease in rye consumption (highest $v$. lowest quartile) seemed smaller for naturally occurring sugar (9-12\%) than for added sugar (21-30\%).

Regarding dietary fat quality, we found only one study in which non-milk extrinsic sugars were associated with lower SFA, MUFA and PUFA intakes ${ }^{(29)}$. However, total sugars or sucrose also seem to have an inverse association with these different fatty acids ${ }^{(13,31,32)}$. In contrast, Dutch adults adhering to the $<10 \mathrm{E} \%$ added sugar recommendation tended to score lower for the SFA of the healthy diet index indicating a higher SFA intake ${ }^{(26)}$. In our analysis, butter (source of saturated fat) intake was $28-32 \%$ higher in the highest added sugar intake quartile compared with the lowest quartile, whereas margarine intake was 18-21\% lower and fish group consumption $25-28 \%$ lower in the highest added sugar intake quartile compared with the lowest quartile. These results were not observed with naturally occurring sugar, rather the 
opposite. Other studies investigating the relationship between sugar types and food consumption indicative of fat quality are few. An American study with 14709 subjects has, similar to our results, shown a negative relationship between added sugar and fish ${ }^{(14)}$. Adults adherent to the $<10 \mathrm{E} \%$ added sugar recommendation were recently found to score higher for the fish component of the Dutch Healthy Diet Index ${ }^{(26)}$. Overall, further studies are clearly needed to determine the relationship between added sugar intake and dietary fat quality.

We used food groups as crude indicators of diet quality. Studies utilising complementary approaches to disentangle the bearing of added sugar in the diet, such as healthy eating indices or dietary patterns, are still few. The US Department of Agriculture reported that with low EI, high added sugar intake was associated with poorer healthy eating indices compared with those with low intakes of added sugar ${ }^{(33)}$. One study, using principal component analysis of 7-d dietary records in British adults, revealed that non-milk extrinsic sugar $\left(\mathrm{E}^{\%}\right.$ ) correlated inversely with the health-conscious dietary pattern ${ }^{(34)}$. On the other hand, overall diet quality was not associated with adherence to added and free sugar recommendations $(<10 \mathrm{E} \%)$ in a very recent Dutch study ${ }^{(24)}$. Hence, further research is needed to fully characterise the relationship between added sugar and dietary quality in the modern, complex food environment. Overall, studies from different countries with divergent food cultures are hard to compare due to divergent methodologies in estimating added sugar intakes ${ }^{(35,36)}$.

Our study is subject to limitations. First, our analyses regarding sugar intakes in relation to nutrient intake and food consumption were adjusted for several confounders. Despite this, residual confounding may remain. Second, general difficulties in accurately assessing dietary intakes of freeliving individuals apply. This phenomenon reaches far beyond energy-under-reporting, which was taken into account in our analyses. Sugar intake is prone to selective under-reporting, especially among subjects with high $\mathrm{BMI}^{(37)}$. In contrast, fruit and vegetable intakes are likely to be over-reported, thus spuriously increasing the intake of naturally occurring sugar. These viewpoints may explain why added sugar was associated with decreasing waist circumference and BMI in our cross-sectional setting. However, prospective cohort studies, with repeated measurements, are needed to more reliably assess the long-term relationship between added sugar and obesity outcomes. Moreover, the SSB exposure used in most of the studies showing a relationship between added sugar and obesity ${ }^{(1,3)}$ should be complemented with other sugar exposures.

Overall, the quantification of the sugar exposures includes inaccuracy. In Finland, sucrose and fructose cover on average 76 and $73 \%$ of total sugars (all mono- and disaccharides excluding lactose) in women and men, respectively. Therefore, the naturally occurring and added sugar terms in our analysis are only approximations of the true intakes and it remains uncertain how the inclusion of the other saccharides (e.g. glucose, galactose and maltose) may have affected the results. Generally, the quality of food composition databases in the coverage of all monosaccharides remains uncertain and calls for efforts in compilation of food composition information. Without this information, reliable monitoring of added sugar intake is unfeasible.

Our added sugar definition is deviant from the free sugar definition used by the $\mathrm{WHO}^{(5)}$. This was, however, reasonable due to the overall moderate consumption of fruit juice in our study population $(101 \mathrm{~g} / \mathrm{d}$ in women and $142 \mathrm{~g} / \mathrm{d}$ in men). Moreover, the Nordic nutrition recommendations use an added sugar definition similar to our analysis ${ }^{(6)}$. In addition, increasing added sugar intake was not associated with fruit juice consumption in this study, which suggests that high added sugar consumers not necessarily are high consumers of fruit juice.

Furthermore, a total of $2.9 \%$ of the subjects had missing data in one or more components forming the naturally occurring sugar variable. However, excluding these subjects from the main analyses (nutrient and food group association analyses) did not affect the results (data not shown). Impurity of the sugar estimates results also from the calculation procedure. Certain food items (e.g. industrial milk products including real fruits and canned fruits and vegetables, with added sugar) are not disaggregated, and thus some naturally sugarcontaining foods may include small amounts of added sugar and vice versa. This was true only for a small proportion of the FFQ food items and the effect on subject misclassification is therefore considered small.

To conclude, this study suggests that naturally occurring and added sugars are associated with divergent food consumption habits, which should be taken into account when studying sugar in relation to health outcomes. A uniform methodology to assess added sugar intake would benefit such efforts and ease cross-study comparisons. The results may inform stakeholders in improving sugar-related communication to foster population health.

\section{Acknowledgements}

N. E. K. performed the statistical analyses and wrote the manuscript. M. E. S. and S. M. contributed to the original conception of this study. M. E. S., N. K. and S. M. contributed to the interpretation of the results. L. M. V. and S. M. contributed to the design and data acquisition of the DILGOM study. K. H. assisted with the statistical analyses. All authors read and approved the final manuscript.

The present study was supported by the Doctoral Programme in Population Health, University of Helsinki; The Finnish Cultural Foundation; and the Juho Vainio Foundation. The DILGOM study was funded by the Academy of Finland (S. M., grant numbers 136895 and 263836).

The authors have no financial or personal conflicts of interest to declare.

\section{References}

1. Te Morenga L, Mallard S \& Mann J (2012) Dietary sugars and body weight: systematic review and meta-analyses of randomised controlled trials and cohort studies. BMJ 346, e7492. 
2. Malik VS, Popkin BM, Bray GA, et al. (2010) Sugar-sweetened beverages and risk of metabolic syndrome and type 2 diabetes: a meta-analysis. Diabetes Care 33, 2477-2483.

3. Malik VS, Pan A, Willett WC, et al. (2013) Sugar-sweetened beverages and weight gain in children and adults: a systematic review and meta-analysis. Am J Clin Nutr 98, 1084-1102.

4. Huang C, Huang J, Tian Y, et al. (2014) Sugar sweetened beverages consumption and risk of coronary heart disease: a meta-analysis of prospective studies. Atherosclerosis 234, 11-16.

5. World Health Organization (2015) Guideline: Sugars Intake for Adults and Children. Geneva: World Health Organization.

6. Nordic Council of Ministers (2014) Nordic Nutrition Recommendations 2012. Integrating Nutrition and Physical Activity, 5th ed. Copenhagen, Denmark: Norden.

7. Cummings JH \& Stephen AM (2007) Carbohydrate terminology and classification. Eur J Clin Nutr 61, S5-S18.

8. Livingstone MB \& Rennie KL (2009) Added sugars and micronutrient dilution. Obes Rev 10, Suppl. 1, 34-40.

9. Sadler MJ, McNulty H \& Gibson S (2015) Sugar-fat seesaw: a systematic review of the evidence. Crit Rev Food Sci Nutr 55, 338-356.

10. Bolton-Smith C \& Woodward M (1994) Dietary composition and fat to sugar ratios in relation to obesity. Int J Obes Relat Metab Disord 18, 820-828.

11. Bolton-Smith C \& Woodward M (1995) Antioxidant vitamin adequacy in relation to consumption of sugars. Eur J Clin Nutr 49, 124-133.

12. Baghurst K, Baghurst P \& Record S (1992) Demographic and nutritional profiles of people consuming varying levels of added sugars. Nutr Res 12, 1455-1465.

13. Cobiac L, Record S, Leppard P, et al. (2003) Sugars in the Australian diet: results from the 1995 National Nutrition Survey. Nutr Diet 60, 152-173.

14. Bowman SA (1999) Diets of individuals based on energy intakes from added sugars. Fam Econ Nutr Rev 12, 31-38.

15. Vartiainen E, Laatikainen T, Peltonen M, et al. (2010) Thirty-five-year trends in cardiovascular risk factors in Finland. Int J Epidemiol 39, 504-518.

16. Konttinen H, Männistö S, Sarlio-Lähteenkorva S, et al. (2010) Emotional eating, depressive symptoms and self-reported food consumption. A population-based study. Appetite 54, 473-479.

17. Kaartinen NE, Tapanainen H, Valsta LM, et al. (2012) Relative validity of a FFQ in measuring carbohydrate fractions, dietary glycaemic index and load: exploring the effects of subject characteristics. Br J Nutr 107, 1367-1375.

18. Reinivuo H, Hirvonen T, Ovaskainen ML, et al. (2010) Dietary survey methodology of FINDIET 2007 with a risk assessment perspective. Public Health Nutr 13, 915-919.

19. Tolonen H, Koponen P, Aromaa A, et al. (editors) (2008) Recommendations for the Health Examination Surveys in Europe. Report no.: B21/2008. Helsinki: National Public Health Institute of Finland.

20. Willett W \& Stampfer MJ (1986) Total energy intake: implications for epidemiologic analyses. Am J Epidemiol 124, 17-27.
21. World Health Organization (1985) Energy and Protein Requirements. Report of a Joint WHO/FAO/UNU Expert Consultation. WHO Technical Report Series no. 724. Geneva: World Health Organization.

22. Goldberg GR, Black AE, Jebb SA, et al. (1991) Critical evaluation of energy intake data using fundamental principles of energy physiology: 1. Derivation of cut-off limits to identify under-recording. Eur J Clin Nutr 45, 569-581.

23. Berrigan D, Dodd K, Troiano RP, et al. (2003) Patterns of health behavior in U.S. adults. Prev Med 36, 615-623.

24. Prendergast KB, Mackay LM \& Schofield GM (2016) The clustering of lifestyle behaviours in New Zealand and their relationship with optimal wellbeing. Int J Behav Med 23, 571-579.

25. Parnell W, Wilson N, Alexander D, et al. (2008) Exploring the relationship between sugars and obesity. Public Health Nutr 11, 860-866.

26. Sluik D, van Lee L, Engelen AI, et al. (2016) Total, free, and added sugar consumption and adherence to guidelines: The Dutch National Food Consumption Survey 2007-2010. Nutrients 8, 70.

27. U.S. Department of Health and Human Services and U.S. Department of Agriculture (2015) 2015-2020 Dietary Guidelines for Americans, 8th ed. http://Health.Gov/Dietaryguidelines/2015/ Guidelines/ (accessed May 2016).

28. Emmett PM \& Heaton KW (1995) Is extrinsic sugar a vehicle for dietary fat? Lancet 345, 1537-1540.

29. Gibson SA (1996) Are high-fat, high-sugar foods and diets conducive to obesity? Int J Food Sci Nutr 47, 405-415.

30. Marriott BP, Olsho L, Hadden L, et al. (2010) Intake of added sugars and selected nutrients in the United States, National Health and Nutrition Examination Survey (NHANES) 2003 2006. Crit Rev Food Sci Nutr 50, 228-258.

31. Gibney M, Sigman-Grant M, Stanton JL Jr, et al. (1995) Consumption of sugars. Am J Clin Nutr 62, 1 Suppl., 178S-193S; discussion 194S.

32. Linseisen J, Gedrich K, Karg G, et al. (1998) Sucrose intake in Germany. Z Ernahrungswiss 37, 303-314.

33. Britten P, Basiotis P, Davis CA, et al. (2000) Is intake of added sugars associated with diet quality? A Publication of the USDA Center for Nutrition Policy and Promotion. Nutrition Insights INSIGHT 21. https://www.cnpp.usda.gov/sites/default/files/ nutrition_insights_uploads/Insight21.pdf (accessed January 2017).

34. Gibson S \& Ashwell M (2011) Dietary patterns among British adults: compatibility with dietary guidelines for salt/sodium, fat, saturated fat and sugars. Public Health Nutr 14, 1323-1336.

35. Louie JC, Moshtaghian H, Boylan S, et al. (2015) A systematic methodology to estimate added sugar content of foods. Eur J Clin Nutr 69, 154-161.

36. Newens KJ \& Walton J (2016) A review of sugar consumption from nationally representative dietary surveys across the world. J Hum Nutr Diet 29, 225-240.

37. Johansson L, Solvoll K, Bjorneboe GE, et al. (1998) Under- and overreporting of energy intake related to weight status and lifestyle in a nationwide sample. Am J Clin Nutr 68, 266-274. 